\title{
Xiuyi Zhi: bent on the fight against lung cancer in his 35-year career
}

Submitted Apr 19, 2018. Accepted for publication Apr 25, 2018.

doi: $10.21037 /$ jtd.2018.05.04

View this article at: http://dx.doi.org/10.21037/jtd.2018.05.04

Nowadays, lung cancer is the most common and fatal malignancy in China. The prevention and early diagnosis/ treatment of this disease have been on the list of the government's priorities and became one of the major concerns of the general public. Meanwhile, the past decades have also witnessed the rapid advances in lung cancer early screening technology, artificial intelligence system for small pulmonary nodules, robotic and minimally invasive surgeries, and molecularly targeted therapy. As declared by Charles Dickens in his book A Tale of Two Cities, "It was the best of times, it was the worst of times."

About 35 years ago, the prevalence of lung cancer in China began to rise, whereas that of tuberculosis began to decline. Xiuyi Zhi was just graduated from Capital Medical University and has dedicated himself to surgical treatment of lung cancer since then. Thirty-five years later, he has become a leader in the thoracic surgery and the diagnosis and treatment of lung cancer in China.

At the beginning of 2002, with the support and leadership of Capital Medical University, Prof. Zhi set out to establish the Lung Cancer Diagnosis and Treatment Center of Capital Medical University. With the contributions of the relevant disciplines including thoracic surgery, respiratory medicine, oncology, medical imaging, and pathology from all the affiliated teaching hospitals of Capital Medical University, the center successfully opened in Xuanwu Hospital of Capital Medical University in April 2003. Fifteen years since the establishment of the lung cancer center, Zhi has established a multi-center clinical research collaboration network for lung cancer across China. In addition, he urged to separate thoracic surgery from cardiac surgery; thanks to the support of Beijing Medical Association, he established the Beijing Society of Thoracic Surgery and was elected as the founding chairman of the society (and also the head of the lung cancer group) in 2008. In the same year, together with Prof. Tianyou Wang and other thoracic surgery experts, he established the Chinese Medical Doctor Association Thoracic Surgery
Branch and was elected as the vice president and directorgeneral of the society. Also in 2018, he initiated the establishment of the Lung Cancer Group of the Chinese Society for Thoracic and Cardiovascular Surgery and served as its chief. In May 2013, he established the China Thoracic Surgery-Lung Cancer Cooperative Group, offering a more specialized platform for the academic exchanges and discipline development in the field of lung cancer diagnosis and treatment. Commissioned by the Ministry of Health of China, he chaired the development of China's Primary Lung Cancer Diagnostic and Treatment Practices, Expert Consensus on Perioperative Airway Management in the Department of Thoracic Surgery, and Expert Consensus on A Multidisciplinary Approach to Perioperative Airway Management, which have promoted the standardized management of lung cancer and multidisciplinary collaboration in China. As the vice president of Chinese Association on Tobacco Control, director of awarenessraising Department of Chinese Anti-cancer Association, and director of Tobacco Control and Lung Cancer Prevention and Control Department of Cancer Foundation of China, he has spared no effort to publicize popular knowledge about tobacco control and lung cancer prevention in the past two decades. He is also the vice chairman of the Central Committee of Health Care of the Chinese Peasants' and Workers' Democratic Party, representative of the 11th Beijing Municipal People's Congress, and special inspector of the Beijing municipal government. From the government level, he strives to promote tobacco control legislation and standardize the diagnosis and treatment of lung cancer.

For decades, Prof. Zhi has devoted his life to lung cancer prevention and treatment in China. "I bave many academic titles from many institutions, and all of them are closely related to the prevention and treatment of lung cancer." said Prof. Zhi. "Being a lung cancer doctor, I must do it perfectly well."

This was where he started and has never changed. Success comes from concentration and perseverance. That's 


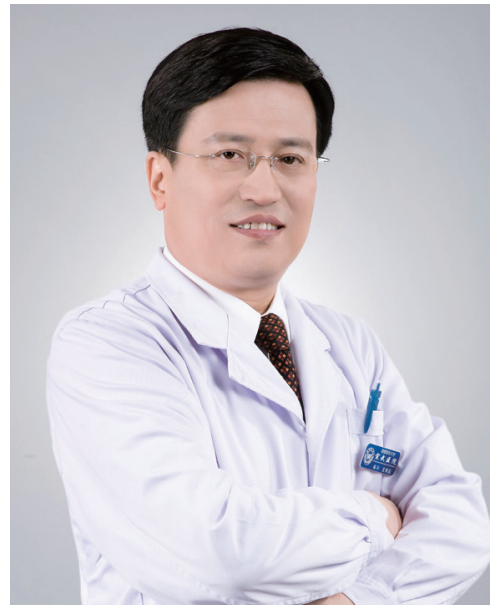

Figure 1 Prof. Xiuyi Zhi.

also a true story for Prof. Zhi during his persistent efforts in helping patients fight against lung cancer (Figure 1).

\section{Expert's introduction}

Prof. Xiuyi Zhi, Director, Department of Thoracic Surgery, Xuanwu Hospital of Capital Medical University; Director, Lung Cancer Center of Capital Medical University; Chairman, China Thoracic Surgery-Lung Cancer Federation; Vice president of Chinese Association on Tobacco Control \& Chairman of Lung Cancer Professional Committee; Standing Director of China International Exchange and Promotion Association for Medical and Healthcare \& Chairman of Lung Cancer Prevention and Control Branch; Vice chairman of Thoracic Surgery Branch of China International Exchange and Promotion Association for Medical and Healthcare \& Chief of Lung Cancer Group; Vice Chairman of the Association for Medical Technology Application of Chinese Hospital Association (MTA); Director, Tobacco Control and Lung Cancer Prevention and Control Department of Cancer Foundation of China; Director of Awareness-raising Department of Chinese Anti-cancer Association; Vice Chairman of Science Popularization Branch of Chinese Medical Doctor Association; Vice President, Beijing Medical Award Foundation; Executive vice-chairman of Beijing Health Education Association; Founding Chairman of Beijing Medical Association Thoracic Surgery Branch; Vice Editor-in-Chief of Chinese Fournal of Thoracic Surgery; Vice Editor-in-Chief of Chinese Medical fournal Frontiers; Vice Editor-in-Chief of Chinese Fournal of Clinical Thoracic and Cardiovascular Surgery; Chief of the Expert Panel of Guidelines on the Diagnosis and Treatment of Primary Lung Cancer (by National Health and Family Planning Commission); Chief of the Expert Panel of Thoracic Surgery, Ministry of Health Expert Committee on Clinical Pathway Review; Chairman of Editorial Committee, Chinese Contemporary Classic Surgeries (Volume of Thoracic Surgery).

\section{A doctor tagged with lung cancer}

If you search "Xiuyi Zhi" in Chinese search engine Baidu, there are 1,070,000 million results; if you try "lung cancer; Xiuyi Zhi", there are still 960,000 results. Intuitively, the name of "Xiuyi Zhi" is tightly related with "lung cancer". "I am a thoracic surgeon with a tag of 'lung cancer'." Prof. Zhi admitted frankly during the interview. A series of his work done on lung cancer has become a strong support for this tag.

October 2002 was a turning point for Prof. Zhi in his career. After he returned from Miami, USA, he left Beijing Tuberculosis \& Breast Tumor Hospital and joined Xuanwu Hospital of Capital Medical University as the director of the Department of Thoracic Surgery. Based on the advanced concepts of "multidisciplinary cooperation" and "multi-center collaborative lung cancer treatment mode", he set out to establish the "Capital Medical University Lung Cancer Diagnosis and Treatment Center".

At that time, the Department of Thoracic Surgery in Xuanwu Hospital was only a professional group under the Department of Thoracic and Cardiovascular Surgery \& Urology, with only two doctors and several beds, and only dozens of surgeries were performed annually. Under the strong support of Dr. Zhang Jian, the then Director of Xuanwu Hospital, the Department of Thoracic Surgery was gradually separated from the Department of Thoracic and Cardiovascular Surgery \& Urology. More talents joined the department, and many sophisticated equipments including video-assisted thoracoscope, mediastinoscope, and ultrasound bronchoscopy were introduced. The department put on an entirely new look thanks to the preferential policies of the hospital and a series of measures taken in the department. "The annual number of surgeries were soon doubled or tripled", said Zhi (Figure 2).

In April 2003, under the leadership of Capital Medical University and Xuanwu Hospital, Prof. Zhi advocated and organized the establishment of "Capital Medical University Lung Cancer Diagnosis and Treatment Center", which was 


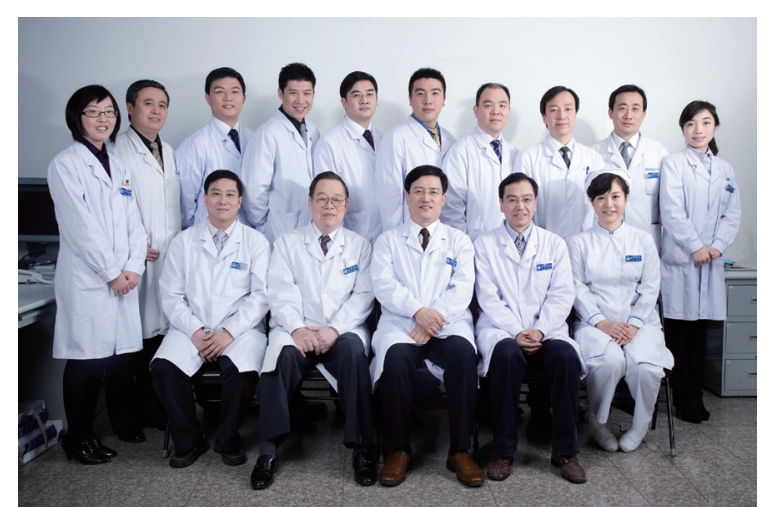

Figure 2 A group picture of the Department of Thoracic Surgery, Xuanwu Hospital.

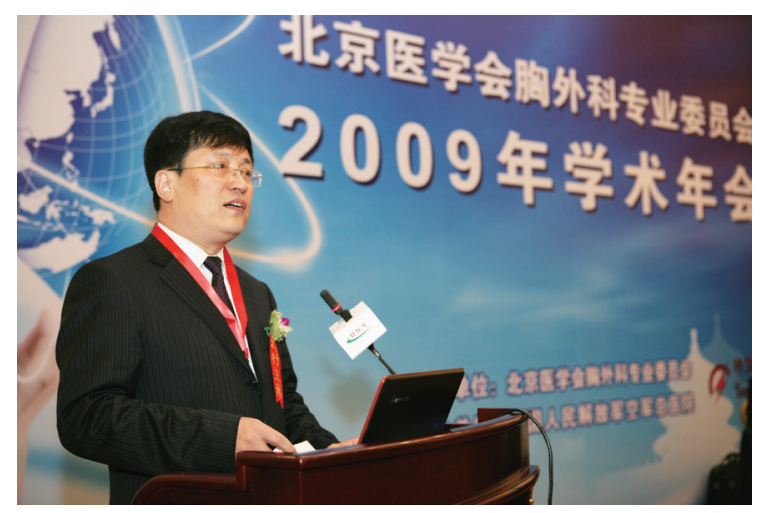

Figure 3 In August 2008, under the direct leadership of Beijing Medical Association, the Professional Committee of Thoracic Surgery of Beijing Medical Association was established. Prof. Zhi was elected as the chairman of the committee and also the head of the Lung Cancer Research Group.

steered by the Department of Thoracic Surgery of Xuanwu Hospital and participated by the Department of Respiratory Medicine, Department of Medical Imaging, Department of Pathology, Department of Nuclear Medicine, Pain Center, and PET Center of Xuan Hospital and by the departments of thoracic surgery and other related departments in Capital Medical University-affiliated Beijing Friendship Hospital, Anzhen Hospital, Chaoyang Hospital, Tongren Hospital, Tiantan Hospital, Electric Power Hospital, and Fuxing Hospital. On this new platform, Prof. Zhi led clinical multicenter studies on preoperative neoadjuvant chemotherapy and postoperative adjuvant chemotherapy for stage III non-small cell lung cancer (NSCLC); by using PET and SPECT combined with video-assisted mediastinoscopy, video-assisted thoracoscopy, and ultrasound bronchoscopy, he carried out multidisciplinary clinical studies on preoperative and pre-treatment clinical staging of lung cancer; also, he actively advocated and promoted the standardized diagnosis and treatment of NSCLC and established a nationwide multi-center clinical research collaboration network for the multidisciplinary treatment of lung cancer. This was a new attempt made by Prof. Zhi. Also, it represented a new mode for lung cancer diagnosis and treatment in China.

"When I came to Xuanwu Hospital, I had a dream to establish a division of lung cancer in the general thoracic surgery department and create a thoracic surgery-lung cancer program that can be a ballmark in Beijing and even across China." said Prof. Zhi, "I feel proud that our efforts have become quite fruitful."

In the community of thoracic and cardiovascular surgeons, Xiuyi Zhi is a famous "separatist", who spares no effort to promote the separation of "thoracic surgery" from "cardiac surgery". For Prof. Zhi, the development of general thoracic surgery in China depends highly on professionalism and teamwork. In this new era, only the separation can win more developmental space for thoracic surgery.

In 2007, thanks to the efforts of Prof. Zhi, the Thoracic Surgery Group (and later the Department of Thoracic Surgery) was established in the Department of Thoracic and Cardiovascular Surgery of Capital Medical University. In 2007, the Chinese Association of Thoracic Surgeons was established. In 2008, the Beijing Society of Thoracic and Cardiovascular Surgery was re-constructed as the Beijing Society of Thoracic Surgery (Figure 3). For more than 10 years, he successfully planned and hosted eight sessions of China North-South Summit Forum on Lung Cancer, 14 sessions of National Thoracic Surgery Directors' Summit on Lung Cancer, 14 sessions of State-level Continuing Education Program-National Workshop on New Advances in Technologies for Lung Cancer Diagnosis and Treatment, six sessions of China-Japan-South Korea Symposium on Lung Cancer, three sessions of Crossstrait Symposium on Tobacco Control and Lung Cancer Prevention, and other well-known academic conferences in China. "I tend to deal with each detail for both scientific research and meeting agenda. I personally participated in the planning and scheduling of each forum and seminar. Sometimes I worked overtime until late at 2 am to amend the meeting program." Zhi smiled. "After the platforms were set up one after another, we have more spaces to exchange our opinions, which allow us to 
make joint efforts in lung cancer prevention, early diagnosis, and early treatment."

In addition, in order to raise the general public's awareness of lung cancer and achieve the early prevention, early diagnosis, and early treatment of this malignancy, Prof. Zhi spends much of his spare time delivering knowledge on tobacco control and lung cancer prevention. In the media, he uses many easy-to-understand words and phrases such as "lung cancer is a disease from 'gas", "away from polluted air, away from lung cancer", and "leave a photo of your lungs with chest CT".

With more reputation and titles, Prof. Zhi becomes more famous, and a chorus of voices began to question him as "a showman". He responded calmly, "No matter how many academic titles I have in my life, they are closely related to lung cancer. Only when you are in a specific position can you have the ability and resources to do a little bit for the development of thoracic surgery and for the prevention and treatment of lung cancer."

"I always hope that I can contribute to tobacco control and lung cancer prevention and treatment in China by building professional technology platforms, training young talents, developing new technologies, establishing professional societies, and creating well-recognized forums. I hope this industry can become bigger and stronger, radiating from Beijing to China, Asia, and the whole world!" According to Prof. Zhi, although the tobacco control and lung cancer prevention and treatment have made great progress with the joint efforts of colleagues across China, there is still a long way to go.

\section{Left, or right?}

"Why are you interested in lung cancer?" we asked. Prof. Zhi did not answer directly; rather, he told us some stories about choice.

Like many other doctors, Zhi decided to be a doctor for his family. When he was young, his mother was not in good health. She often visited a local hospital and took medicine almost every day. Thus, he was determined to do medicine. In the eyes of young Zhi, " $A$ man should be surgery if he chooses medicine as his profession. This may be a common sense among most male medical students." What's more, the father of a classmate was just the director of the department of thoracic surgery in a large hospital in Beijing. "Thoracic surgery" thus became the most appealing discipline for him.

In 1983, the 24-year-old Xiuyi Zhi graduated from the Beijing Second Medical College (now the Capital Medical University) and was assigned to the Tongxian County-based
Beijing Tuberculosis and Lung Cancer Institute \& Hospital ("Tuberculosis Hospital"; now the Beijing Chest Hospital, Capital Medical University). At that time, the Tuberculosis Hospital was the most famous thoracic hospital in China, and Professor Yuling Xin, a well-known thoracic surgeon in China, was the founder of the Department of Thoracic Surgery in this hospital. Under his careful instructions, a large number of nationally renowned thoracic surgeons including Lianfu Cai, Zhiwen Zhao, Dongling Zuo, Xinsun Qiu, Qibang Hu, and Bingsheng Ge emerged from the Tuberculosis Hospital. The first case of human lung transplantation in China was also completed in this hospital. "I will be another top thoracic surgeon in this department," Zhi was full of hope.

However, things didn't happen quite that way. Since Zhi had an excellent curriculum (for instance, he had served as a student cadre from elementary school to university), the human resource department of the Tuberculosis Hospital planned to enroll him in the management team. There came a chance: Prof. Baoyu Xie, a famous radiologist and also the director of the Department of Radiology, had been nearly 60 years old and was about to retire. The hospital leaders and the human resource department hoped that Zhi could be working with Prof. Xie and then took his position a few years later. In this way, he would have been the youngest director of a radiology department in China. However, Xiuyi Zhi did not hesitate to refuse such a good opportunity.

"I want to be a surgeon. I came to the Tuberculosis Hospital to be a thoracic surgeon. Performing surgeries as a thoracic surgeon is my only goal." said Zhi. A "cold war" broke out between young Zhi and the hospital. He firmly refused any position in the Radiology Department. The "war" was deadlocked for nearly a month and ended with the compromise of the human resource department. Finally, Zhi entered the Department of Thoracic Surgery as he wished.

Since the department had not recruited new surgeon for 14 years, Zhi was carefully instructed by many senior surgeons and got a lot of opportunities for clinical practice. Every day was a cheerful day for him. Top thoracic surgeons including Xinsun Qiu, Lijin He, and Meiyun Lin brought him to operate on patients on a daily basis, which is unimaginable for a new resident today. As the only resident in the Department of Thoracic Surgery, Zhi was responsible for the daily management of 30-40 inpatients in the ward, which made him grow fast.

Just 3 years later, Zhi got a precious chance to receive further training in Japan. Quite unexpectedly, he had to face 
another choice. After he successfully passed the English Proficiency Test (EPT) following half a year's off-the-job training, he was told that he must be trained on tuberculosis testing in Japan. Prof. Biya Yan, a famous TB specialist in China and the then director of Beijing Tuberculosis Research Institute, advised him, "Xiuyi, Japan has the most advanced tuberculosis testing technology, and our hospital also needs young talents like you in this field. Hopefully you can cherish this training chance." Deputy institute director Duanmu, who was responsible for scientific research, and the chief of the human resource department also talked to him, expressing their high expectations for you. They hoped that Zhi could follow the hospital's arrangement to learn laboratory tests in Japan for 1 year and then return home to become the new director of the clinical laboratory. At that time, Zhi not only devoted himself to the thoracic surgery but also had locked his professional direction in lung cancer. It was exciting to go abroad for further study and to be a successor of the director of the clinical laboratory; for Zhi, however, it was definitely unacceptable to him if he had to say bye-bye to thoracic surgery. So, he once again said no to this training chance.

Later, there came another good chance: to study tuberculosis diagnosis and treatment technology in Netherlands and later work in the National Tuberculosis Center. Again, Zhi refused because this meant that he had to leave the Department of Thoracic Surgery. So far, he had given up three chances to change his professional direction. Of course, he felt sorry for the leaders of the hospital. "In these years, young doctors could come home only once a week. After making his rounds in the hospital on Sunday morning, I went to the Xinbua Street to take the Route 332 bus to go home; or, I would ride for longer than one hour before I could reach home. After all that hard work, I just wanted to be a thoracic surgeon. I was really curious why the hospital leaders always tried to promote me by asking me to leave the Department of Thoracic Surgery." Zhi He sighed as he recalled this memory.

By the end of 2007, Zhi had been working in the Department of Thoracic Surgery of Xuanwu Hospital for 4 years; once again, he was faced with another tough decision in life. At that time, the position of the executive vice president and secretary-general of Beijing Medical Association was vacant. Mr. Dapeng Jin, the then PartySecretary and Director of Beijing Municipal Health Bureau, appreciated Zhi's strong management capability and wanted Zhi to take this very important position. This motion was immediately approved by the party committee of Beijing Health Bureau. However, the premise of this appointment was Zhi's resignation of his positions including the director of the Lung Cancer Center of Capital Medical University and the director of Department of Thoracic Surgery in Xuanwu hospital. Zhi hesitated, then declined this kind arrangement, which also meant the door of working as a public servant closed for him.

"So you can easily understand why I'm so attached to lung cancer. Since I bave been a surgeon for lung cancer for so many years and I have refused so many great opportunities for this target, I must do it perfectly well." Zhi smiled.

\section{Introduction and localization of ERAS in China}

According to Zhi, thoracic surgery in China is facing great challenge. According to the current disease spectrum in China, the incidence of lung cancer will not come down until 2030. "We have the largest lung cancer population and the largest amount of surgery worldwide. We should remain true to our original aspiration and keep our mission firmly in mind, making our voices heard in international venues and across the world." Enhanced recovery after surgery (ERAS) is an indispensable part of it.

ERAS was first proposed by Danish surgeon $\mathrm{H}$. Kehlet in the 1990s. In 2005, it was introduced into China. As an innovative perioperative care mode, it advocates for a series of new measures to optimize perioperative management and treatment and is designed to achieve early recovery after surgical procedures by reducing pain and risk during the operation.

"You will not know bow painful a patient is before you get sick." In the past 5 or 6 years, Zhi has suffered from Achilles tendon rupture and undergone an eye surgery. "When I got sick, I personally experienced various inconveniences and could understand patients better. Actually my problems were not so serious. I can imagine the pain and inconvenience experienced by a patient who has had a major chest surgery." Unlike the conventional treatment modes, ERAS shifts the focus of treatment from "disease" to "patient", paying more attention to the quick physical and mental recovery, so as to shorten hospital stay, reduce medical expenses, and improve postoperative quality of life. "Every day a large number of patients from other provinces and cities are seeking treatment in about 60 tertiary hospitals in Beijing. Their longer hospital stay means their families' longer stay in hotels. If these patients recover earlier from a surgery, they will be able to go home with their families earlier."

Airway management is an important component of ERAS in the department of thoracic surgery. Effective 
airway management and lung protection measures can lower the incidences of perioperative airway complications. Early in 2009, the Chinese Society for Thoracic and Cardiovascular Surgery and the Chinese Association of Thoracic Surgeons released the "Expert Consensus on Perioperative Pulmonary Protection during Thoracic Surgery", which proposed a series of perioperative lung protection measures to prevent the occurrence of severe lung complications. In 2012, led by Zhi, a group of thoracic surgeons developed the "Expert Consensus on Perioperative Airway Management in the Department of Thoracic Surgery", which has strongly promoted the clinical application of perioperative airway management in departments of thoracic surgery in China.

"This document was compiled by thoracic surgeons. All the sections related with respiratory medicine and anesthesiology were written by thoracic surgeons." With the interpretation and promotion of the expert consensus, Zhi has increasingly recognized that perioperative airway management is not just a matter of the department of thoracic surgery; rather, it covers pre- and post-operative care, pain management, emotional management, and nutrition and relevant departments including department of respiratory medicine, department of anesthesiology, postoperative care unit, and nursing department must be involved.

For example, the postoperative wound pain can cause skeletal muscle tone, and the cough and expectoration can be affected due to the fear of pain, resulting in lower thoracic and lung compliance. In the past few years, Zhi has a strong feeling that ERAS is in a forgotten corner. If the postoperative wound pain is not properly managed, patients dare not to cough up sputum. As a result, atelectasis can occur. Inadequate ventilation leads to a decrease in tidal volume and vital capacity, resulting in anoxia and carbon dioxide accumulation. Meanwhile, pain can restrict the patients to change their body position; as a result, the airway secretions can not be effectively discharged, which increases the opportunity of pulmonary infection.

Improper placement of drainage tube is the most common cause of postoperative pain. "While we ourselves bave not been a patient in the department of thoracic surgery, we can imagine how painful the patients are when the drainage tube pokes the diaphragm and the lung tissues. They will not dare to cough when they feel severe pain even during normal breathing." One of Zhi's colleagues suffered from such pain. After having received a lung surgery, he could not tolerate the pain caused by the drainage tube and removed it by himself that night. When the colleague talked about this story, he said, "You don't even know how much it hurts!" Zhi was deeply impressed. "Patients have varying abilities to tolerate pain. A doctor must effectively communicate with their patients before and after surgery. He/she must really care about the patients' feelings after surgery and bring the patients more clinical benefits."

The Expert Consensus on Multidisciplinary Perioperative Airway Management (2016 Edition) has stressed the importance of "tailored treatment" for postoperative analgesia and advocates for multimode analgesia. Prof. Zhi told us that the expert group would update the expert consensus this year based on the currently available evidences, especially in the aspects including pain management, psychological counseling, and nutritional support.

During the development of guidelines and expert consensuses, we are constantly optimizing the contents of ERAS, especially the localization of this concept. During its application in clinical practices, we constantly look for new evidences and enrich it with new elements. The multidisciplinary cooperation mode is gradually formed in this process. For example, the importance of a nursing team in ERAS cannot be overemphasized. In the expert consensus on perioperative airway management, tobacco cessation counseling, preoperative atomization, postoperative sputum evacuation, and psychological care are done by a nursing team. We must fully recognize the importance of nursing team in ERAS. In 2009, Beijing Society of Thoracic Surgery specially established the Academic Salon of the Head Nurses in Thoracic Surgery Departments in Beijing, which gradually evolved into the Beijing Annual Meeting of Thoracic Nursing. With the establishment of multi-disciplinary platforms, spread of ERAS concept, and construction of thoracic surgery subdisciplines, the nursing teams are playing increasingly important roles in the psychological management, high-quality nursing, and management throughout ERAS.

"Although it initially was a foreign concept, ERAS now has been localized in China. China has a buge population of patients, and the 'real-world' clinical practice and big data must be generated in China." Zhi said decisively.

\section{Talents and platforms: the bases for a new era of ERAS in thoracic surgery}

With the localization of ERAS in China, "the most important thing now is to train talents and establish platforms, so that the whole surgery community can work together to create a better environment." 
At the end of 2016, the first West China Forum on ERAS in Thoracic Surgery was held in Chengdu, Sichuan Province. A questionnaire-based survey performed in the meeting showed that up to $69.6 \%$ of doctors and $58.7 \%$ of nurses believed that ERAS was still a concept rather than a practice in clinical settings. This result was not surprising for Zhi. He explained that the clinical practice and discipline construction of ERAS were carried out only in Beijing, Tianjin, Shanghai, Guangzhou, Chongqing, and other large cities or in some university-affiliated hospitals, and centers with specialized subdisciplines and teams under a specific system account for only 10-20\%. "Ten years ago, the introduction of the ERAS concept opened a window for Chinese surgeons; today, the release and interpretation of the Expert Consensus opened a door. Howvever, we are still outside the room."

Then, how can thoracic surgery in China formally enter an era of ERAS? Shi put forward some suggestions.

First of all, we should further spread the idea of ERAS and promote the use of the Chinese and foreign expert consensuses among thoracic surgeons. ERAS has not been well integrated in the continuing education program, and this new concept may be effectively promoted via new communication modes. When an annual meeting of thoracic surgery is held at a university hospital or by an association/society, the leaders of the universities and hospitals should be informed about ERAS. In any academic meeting of thoracic surgery, the department directors should be educated about ERAS. In particular, we should actively seek academic leaders in university hospitals who are interested in the development of ERAS.

Secondly, we should carefully summarize the clinical experiences in hospitals that apply ERAS, timely update the existing diagnosis/treatment guidelines and expert consensuses, and actively create platforms for academic exchange and project cooperation. On June 23, 2017, the ERAS Branch of China International Exchange and Promotion Association for Medical and Healthcare was formally established. This is the only secondary society named after ERAS in China. Through such an academic platform, we may constantly create model centers that allow the medical staff from different hospitals to share and exchange their experiences.

In addition, ERAS is a pipeline. It is far beyond the intra-hospital workflow; in fact, community hospitals and community medical service centers, and home care also play important roles. For instance, according to the Expert Consensus on Multidisciplinary Perioperative Airway Management (2016 Edition), "for patients at bigh risk for postoperative pulmonary complications, aerosolized corticosteroids in combination with bronchodilators 3-7 days before surgery and 3-7 days after surgery can significantly reduce the risk of postoperative pulmonary complications." However, restricted by the current performance evaluation indicators in hospitals, the current guidelines and expert consensuses often can not be adequately implemented within the required hospitalization days. In contrast, if a large provincial hospital can establish a hierarchical health care system or a cooperative mode with nearby community hospitals and secondary hospitals, it will be able to properly implement the perioperative management according to ERAS protocol. In addition, training on people (e.g., family members, nannies, and nursing workers) who support the patients must also be incorporated into the concept of holistic ERAS.

Finally, Zhi hope more thoracic surgeons will actively seek funding support for scientific research on ERAS, publish more ERAS-related articles in peer-reviewed journals, and enroll more graduate students in this subdiscipline. Data from multi-center collaborative studies will greatly affect the societies, which will influence the government in a collaborative way. The government authorities will thus formulate new charge policy and performance evaluation policy and try to persuade the society and general public via awareness-raising campaigns. In this way, we may really enter the ERAS era.

"China has a buge population of lung cancer patients and should make more contributions to ERAS." said Zhi. The establishment of the ERAS Branch of China International Exchange and Promotion Association for Medical and Healthcare has offered a platform for academic exchanges and project cooperation. With the growth of expert teams, establishment of multidisciplinary collaboration modes, and accomplishment of new research projects, more clinical results will be obtained and our voices will be heard worldwide.

\section{Epilogue}

Zhi is 59 years old this year, but he still travels around all over the country for creating academic platforms and spreading medical knowledge. Still he would happily work until 2:00 am for a specific project and then get up by 6:00 am and go to work vigorously. "This is the career I had chosen. This is my favorite profession. I am just so happy, no matter bow much time and energy I have to spend on it!" said Zhi,

"Thoracic surgery in China is in a golden time. With the 
development of socioeconomic status, advances in medical sciences, aging of population, and changes in disease spectra in China, the Chinese surgeons have the obligation and ability to make our voices beard throughout the world, especially in the fields of thoracic surgery and lung cancer." "However, there are still far too few Chinese doctors who are fully bent upon lung cancer!" Zhi signed. What he wants to do now is to create a platform and start an era that will help more young people interested in lung cancer to do more meaningful things.

In fact, if a person does only one thing in his whole life, he may actually achieve great success in his career. If I had performed surgeries for a variety of conditions such as tuberculosis, lung transplantation, and esophageal cancer at the very beginning of my career, I would not have been able to be myself today. "I have been bent upon treating lung cancer in my whole life." Zhi pushed his glasses up his nose and smiled. "When lung cancer can be cured, I will be laid off."

\section{Acknowledgements}

We thank Lili Liao and Yuan Yu from AME Publishing Company for their support and assistance for this article.

\section{Footnote}

Conflicts of Interest: The author has no conflicts of interest to declare.

(Science Editor: Chen Gao, JTD, jtd@amepc.org)
Cite this article as: Gao C. Xiuyi Zhi: bent on the fight against lung cancer in his 35-year career. J Thorac Dis 2018;10(Suppl 11):S1322-S1329. doi: $10.21037 /$ jtd.2018.05.04 